\title{
CONTRACTIVE PROJECTIONS AND PREDICTION OPERATORS ${ }^{1}$
}

BY M. M. RAO

Communicated by Jack Schwartz, July 7, 1969

1. Introduction. The purpose of this note is to present some results on characterizations of subspaces of a general class of Banach function spaces (BFS) admitting contractive projections onto them, and to include an application to nonlinear prediction (and approximation) theory.

Let $L^{\rho}$ be the subspace of all measurable scalar functions $f$ on $(\Omega, \Sigma, \mu)$ with $\rho(f)=\rho(|f|)<\infty$, where $\rho(\cdot)$ is a function norm, i.e., a norm with the additional properties

(i) $0 \leqq f_{n} \uparrow \Rightarrow \rho\left(f_{n}\right) \uparrow$, and

(ii) $\rho(\cdot)$ verifies the triangle inequality for infinite sums. Then $L^{p}$ is also complete, called a BFS, (cf. [6] and [4]). It will also be assumed, for convenience, that $0 \leqq f_{n} \uparrow f \Rightarrow \rho\left(f_{n}\right) \uparrow \rho(f)$, the Fatou property. $\rho(\cdot)$ is an absolutely continuous norm (a.c.n.) if for each $f \in L^{p}$, $\rho\left(f \chi_{A_{n}}\right) \rightarrow 0$ for any $A_{n}$ in $\Sigma, A_{n} \downarrow \varnothing$. If $\mathfrak{X}$ is a $B$-space, $L_{\mathfrak{X}}^{\rho}$ is the space of $\mathfrak{X}$-valued strongly measurable functions $f$ on $\Omega$, with $\rho\left(|f|_{x}\right)<\infty$, where $\rho(\cdot)$ is as above. Then $L_{x}^{o}$ is also complete. Finally let $\mathfrak{M}_{x}^{o}$ $=\overline{\mathrm{sp}}\left\{f x: f \in L^{p}, \quad x \in \mathfrak{X}\right\} \subset L_{\mathfrak{x}}^{p}$. A projection is a linear idempotent operator.

The projection problem, stated at the outset, has been first treated for $L^{\rho}=L^{1}$ in [5], and a more detailed consideration of the same case, with $\mu(\Omega)<\infty$, has been given in [2]. If $L^{p}=L^{p}$, also with $\mu(\Omega)<\infty$, it was then considered in [1], and these results were extended for $L^{p}=L^{\Phi}$, the Orlicz spaces, with a.c.n. and $\mu \sigma$-finite, in [10]. The general solution of the problem in the scalar case, and a less general one in the vector case, will be given below.

2. Contractive projections. Let $S \subset L^{p}$ be a closed subspace. If $L^{\rho} \neq L^{2}$, then, as is well known, not every $\delta$ is the range of a bounded projection. The positive solution is given by the following result for $L^{\rho}$-spaces. (An operator $T$ is positive if $T f \geqq 0$ for $f \geqq 0$.)

THEOREM 1. If $(\Omega, \Sigma, \mu)$ is a measure space, let $L^{p}(\Sigma)$ be the $B F S$ defined above. Consider the statements:

(a) $S$ is the range of a (positive) contractive projection in $L^{p}(\Sigma)$.

(b) there is an isometric isomorphism $\Psi: L^{p}(\Sigma) \mapsto L^{p}(\Sigma),(\Psi=i d e n-$ tity) such that

${ }^{1}$ Supported, in part, under the NSF grant GP-8777. 
(i) $\Psi$ (s) is a B-lattice, i.e., a selfadjoint space with real functions forming a lattice, and

(ii) $0 \leqq f_{n} \in \Psi(s), f_{n} \uparrow f, f \in L^{\rho}(\Sigma) \Rightarrow f \in \Psi(s)$.

(c) there is a (positive) isometric isomorphism between some $L^{p}(B)$ on some measure space $\left(S, \leftrightarrow, \mu_{1}\right)$ and $\mathrm{S}$.

(d) same as (c) except "topological equivalence" replaces "isometric isomorphism."

Then one has $(\mathrm{c}) \Rightarrow(\mathrm{a}) \Leftrightarrow(\mathrm{b}) \Rightarrow(\mathrm{d})$. In case $\rho(\cdot)$ also verifies, $\chi_{A} \in L^{\rho}(\Sigma)$ for each $A \in \Sigma$ with $\mu(A)<\infty$, then (a) $\Leftrightarrow$ (c) also holds.

Remark. If $\rho(f)=\int_{0}^{1}|f| d \mu / x$, with $\Omega=[0,1], \mu=$ Leb.meas., then $\rho(\cdot)$ is a function norm, but $\rho\left(\chi_{\Omega}\right)=\infty$. Thus the last condition of the theorem is a restriction on $\rho$. It can be shown easily that $b$ (ii) automatically holds if $\rho$ is an a.c.n., but will be needed otherwise.

This result is proved through several isomorphisms using equivalent measure spaces and the results of [13]. However, for an application of the latter, a first reduction is needed and is provided by the following result which has independent interest.

THEOREM 2. If $L^{\rho}(\Sigma)$ is a BFS on $(\Omega, \Sigma, \mu)$, then there exists a measure space $(S, \Theta, \nu)$ where $S$ is a locally compact space, $B$ is a $\sigma$-field generated by the compact subsets of $S$ and $\nu$ is a measure assigning finite measure for compacts, in terms of which $L^{p}(S, \Theta, \nu)$, or $L^{p}(B)$, is isometrically (and lattice) isomorphic to $L^{p}(\Sigma)$. Moreover each $f$ in $L^{p}(B)$ has a $\sigma$ compact support. If there exists a strictly positive element in $L^{p}(\Sigma)$, then $S$ can be chosen compact, so that $(S, \Theta, \nu)$ is a finite measure space.

If $\mu$ is $\sigma$-finite then a strictly positive element always exists in $L^{\rho}(\Sigma)$ (e.g., a weak unit, cf. [6, p. 153]) and the last part contains this case. This result is proved using a method of proof of $([8$, Theorem 2.1]) and some results of [13]. (See also [3] for the $L^{1}$-case.) With this reduction, the problem of Theorem 1 can be transfered to $L^{\rho}(B)$. Then it can be isometrically embedded in $L^{P}(\tilde{\mathbb{Q}})$ on a localizable measure space $(\tilde{S}, \tilde{\mathbb{B}}, \tilde{\nu})$ where $B$ goes, under an algebraic isomorphism, into a subring of $\tilde{\mathscr{B}},[13$, Theorem 3.4$]$. Then the proof is successively reduced to the case of finite measure space where the methods and ideas of [2] and [10] can be generalized and used. In this way the full result of Theorem 1 is established.

In general there will be many contractive projections onto $\delta$, when one exists. The following gives a uniqueness result.

Proposition 3. Suppose $L^{\rho}(\Sigma)$ is a rotund (= strictly convex) and smooth ( $=$ norm is Gâteaux differentiable) reflexive space on $(\Omega, \Sigma, \mu)$. Then a closed subspace $S \subset L^{\rho}(\Sigma)$ can be the range of atmost one contrac- 
tive projection. If in particular $S=L^{\rho}(B), B \subset \Sigma, a$-field, then there exists a unique positive contractive projection onto $S$, namely the (gen-

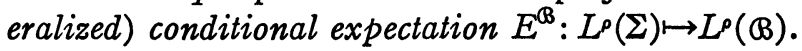

The case of $L^{p}=L^{p}, 1<p<\infty, \mu(\Omega)<\infty$, of the above result was obtained in $([1$, p. 392$])$. The general form of $P$ is not-simple. The following case is illustrative.

PRoposition 4. Let $P: L^{\rho}(\Sigma) \mapsto L^{\rho}(\Theta)$ be a contractive projection (which exists by Theorem 1), where $B \subset \Sigma$ is a $\sigma$-field with $\mu_{\odot} \sigma$-finite, and $L^{p}(\Sigma)$ is a BFS. Then there exists a locally integrable function $g$ such that

(i) $P(\cdot)=E^{\mathbb{B}}(g \cdot)$, and

(ii) $E^{\mathbb{B}}(g)=1$ a.e., where $E^{\mathbb{B}}$ is the conditional expectation relative to $B$.

This shows that while $E^{\beta}$ itself is a contractive projection onto $L^{\rho}(B)$, it is not the general form of the operator. If $\rho$ is an a.c.n., then it can be shown that $g=1$ a.e. here, and this is not necessarily true in the general case. The above two results are proved by an extension of the methods of [10]. A special case of the above proposition for $L^{\Phi}$-spaces, with $\mu(\Omega)<\infty$, was discussed in [11].

For the case of $\mathfrak{T T}_{\mathfrak{x}}^{\rho}$ spaces, the following result holds.

THEOREM 5. Let $L^{\rho}(\Sigma)$ and $\Re_{x}^{\rho}$ be as defined in $\$ 1$. If $\delta \subset L^{p}(\Sigma)$ is a closed subspace, let $\mathrm{s}_{\mathfrak{X}}=\overline{\mathrm{sp}}\{f x: f \in \mathcal{S}, x \in \mathfrak{X}\} \subset \mathfrak{N}_{\mathfrak{x}}^{\circ}$. Also let $\chi_{A} \in L^{\rho}(\Sigma)$ for each $A \in \Sigma$ with $\mu(A)<\infty$. Then the following four statements are equivalent:

(i) $\exists$ contractive projection $P: L^{p}(\Sigma) \mapsto S$.

(ii) $\exists$ contractive projection $P: \mathfrak{N}_{x}^{\circ} \mapsto S x$.

(iii) $\exists L^{p}\left(\bigotimes_{1}, \mu_{1}\right)$, on some measure space $\left(S_{1}, \bigotimes_{1}, \mu_{1}\right)$ and $S$ is isometrically isomorphic to $L^{p}\left(B_{1}, \mu_{1}\right)$.

(iv) $S_{\mathfrak{X}}$ is isometrically isomorphic to $\mathfrak{N}_{\mathfrak{X}}^{o}\left(\bigotimes_{1}, \mu_{1}\right)$.

This result is proved on using Theorem 1 , and the fact that $L^{\rho} \otimes_{\gamma} \mathfrak{X} \subset \mathfrak{N}_{x}^{\circ}$ and is dense in the latter (see [9]). Here $\otimes_{\gamma}$ is the greatest cross-norm, and one then uses a result on projections in crossspaces [12, p. 58]. The general case of $L_{\mathfrak{X}}^{0}$ itself does not seem to follow in this way. The above one already includes the $L_{\mathfrak{x}}^{0}, 1 \leqq p \leqq \infty$ case.

3. Prediction operators. A subspace $M \subset L^{p}$ is said to be a Tshebyshev subspace if for each $x \in L^{p}$ there is a unique $x_{0} \in M$ with $\rho\left(x-x_{0}\right)=\min \{\rho(x-y): y \in M\}$. The operator $P_{M}: x \mapsto x_{0} \in M$, is 
called a prediction operator in nonlinear prediction theory. Though $P_{M}^{2}=P_{M}$, it is not linear in general. If it is linear, the powerful methods of linear analysis will be available in their study. So this is a natural question to treat. If $P_{M}$ is linear, then $Q=I-P_{M}$ is a contractive projection with $M$ as its null space (and the converse also holds). This is the connection between projections and predictions, and a solution can be presented as follows.

THEOREM 6. Let $M \subset L^{p}$ be a Tshebyshev subspace, and $P_{M}$ be the prediction operator for $M$. If $P_{M}$ is linear then the quotient space $L^{p} / M$ is topologically equivalent to $L^{\rho}(B)$ on some measure space $\left(S, B, \mu_{1}\right)$. Conversely, if $L^{p} / M$ is isometrically isomorphic to $L^{p}(B)$ on some $\left(S, \Theta, \mu_{1}\right)$ then $P_{M}$ is linear.

In case $\chi_{A} \in L^{\rho}$ for each $A \in \Sigma, \mu(A)<\infty$, then the above can be stated as: $P_{M}$ is linear $\Leftrightarrow L^{p} / M$ is isometrically isomorphic to an $L^{\rho}(B)$. If $L^{p}=L^{p}, 1<p<\infty, \mu(\Omega)<\infty$, the latter has been obtained in [1]. The general case can be proved quickly with the results of the preceding section. However, it was noted in [10], that for the case $L^{\rho} \neq L^{2}, M$ must be relatively complicated since $P_{M}$ will not be linear if $M$ is of the form $L^{\rho}\left(\Sigma_{1}\right), \Sigma_{1} \subset \Sigma$, a $\sigma$-field.

The proofs of all the results above involve first a characterization of the adjoint space $\left(L^{\rho}\right)^{*}$ of $L^{p}$. This is involved. It is obtained by generalizing the work of ([7] and [4]) appropriately. With these results (and those of [9]), and of [13], the above bare sketch is completed. The details and related results will be published separately.

\section{REFERENCES}

1. T. Ando, Contractive projections in $L^{p}$-space, Pacific J. Math. 17 (1966), 391405.

2. R. G. Douglas, Contractive projections in an $L^{1}$-space, Pacific J. Math. 15 (1965), 443-462.

3. H. W. Ellis, On the dual of $L^{1}$, Canad. Math. Bull. 8 (1965), 809-818.

4. N. E. Gretsky, Representation theorems on Banach functions spaces, Bull. Amer. Math. Soc. 74 (1968), 705-709 (cf., also Mem Amer. Math. Soc. No. 84).

5. A. Grothendieck, Une caractérisation vectorielle-métrique des espaces $L^{1}$, Canad. J. Math. 7 (1955), 552-561.

6. W. A. J. Luxemburg and A. C. Zaanen, Compactness of integral operators in Banach function spaces, Math. Ann. 149 (1963), 150-180.

7. M. M. Rao, Linear functionals on Orlicz spaces: general theory, Pacific J. Math. 25 (1968), 553-585.

8. - Stone-Weierstrass theorems for function spaces, J. Math. Anal. Appl. 25 (1969), 362-371.

9. - Produits tensoriels et espaces des fonctions, C. R. Acad Sci. Paris 268 (1969), 1599-1601. 
10. - Inference in stochastic processes. IV (to appear).

11. Conditional expectations and closed projections, Indag. Math. 27 (1965), 100-112.

12. R. Schatten, $A$ theory of cross-spaces, Ann. Math. Studies, no. 26, Princeton Univ. Press, Princeton, N. J., 1950.

13. I. E. Segal, Equivalence of measure spaces, Amer. J. Math. 73 (1951), 275-313.

MATHEMATISChe INSTITUT DER UNIVERSITÄT, WIEN, AND

Carnegie-Mellon University, Pittsburgh, Pennsylvania 15213 